\title{
IN VIVO LENGTH OSCILLATIONS OF INDIRECT FLIGHT MUSCLES IN THE FRUIT FLY DROSOPHILA VIRILIS
}

\author{
WAI PANG CHAN AND MICHAEL H. DICKINSON* \\ Department of Integrative Biology, University of California, Berkeley, CA 94720, USA
}

Accepted 14 August 1996

\begin{abstract}
Summary
We have used high-speed video microscopy to measure in vivo length oscillations of the indirect flight muscles of the fruit fly Drosophila virilis during tethered flight. The changes in muscle strain were measured by tracking the deformation of the thoracic exoskeleton at the origin and insertion of both the dorsal longitudinal (DLM) and the dorsal ventral (DVM) muscles. The mean peak-to-peak strain amplitudes were found to be $3.5 \%$ for the DLMs and

measurements of indirect flight muscle strain in other insects, but almost an order of magnitude greater than the strain amplitudes used in most biophysical studies of skinned Drosophila fibers. The results suggest that serial compliance within this sarcomere would need to relieve approximately $70 \%$ of the total strain in order for individual crossbridges to remain attached throughout a complete contraction-extension cycle.
\end{abstract} $3.3 \%$ for the DVMs, although the strain amplitude within individual cycles ranged from 2 to $5 \%$. These values are consistent with the small number of previous
Key words: stretch-activation, strain, mechanical power, crossbridge, flight, muscle, Drosophila virilis.

\section{Introduction}

The flight muscles of many insects including beetles, bees and flies are segregated into two anatomically and physiologically distinct classes (for reviews, see Pringle, 1957; Kammer, 1985; Dickinson and Tu, 1996). A set of small twitch muscles insert onto the sclerites of the wing hinge and act to alter wing-stroke kinematics during steering maneuvers (Heide, 1983; Wisser and Nachtigall, 1984; Miyan and Ewing, 1985). The extensive mechanical power required for flight, however, is provided by another group, the dorsal longitudinal (DLM) and the dorsal ventral (DVM) muscles that fill most of the thorax. These specialized power muscles are indirect in that they do not insert directly onto the sclerites of the wing hinge (Pringle, 1957; Snodgrass, 1993). Instead, the successive contractions of the antagonistic DLMs and DVMs deform the thorax laterally and dorso-ventrally to drive the upstroke and downstroke of the wing through a complex mechanical linkage within the wing hinge (Boettiger and Furshpan, 1952; Boettiger, 1957; Miyan and Ewing, 1985; Ennos, 1987; Wisser, 1988). In addition, the power muscles are asynchronous in that individual contractions are not initiated by the presynaptic spikes of the motor neurons (Pringle, 1949; Roeder, 1951). Instead, the muscles are stretch-activated, and the two antagonistic sets function together within the thorax as a self-exciting resonant system (Machin and Pringle, 1960; Aidley, 1985). Both the indirect morphology and asynchronous physiology are specializations that allow these muscles to maximize power output, especially when operating at high frequency (Josephson and Young, 1987; Ellington, 1991).

Although the stretch-activation is central to the flight physiology and thus the evolutionary success of the world's most diverse group of organisms, the biophysical basis of this mechanism is poorly understood. The asynchronous muscles of some flies function at frequencies as high as $1000 \mathrm{~Hz}$ (Sotavalta, 1947), which seems to preclude multiple actin-myosin binding cycles within a single contraction. One possible solution to this enigma is for changes in sarcomere length in stretch-activated muscles to be short enough for crossbridges to remain attached throughout an entire extension-contraction cycle, although still undergoing a forcegenerating conformational change (Alexander, 1995). The feasibility of this model could be tested by accurate measurements of length changes within the muscle during flight. So far, most measurements of in vivo strain of flight power muscles have focused on bees. Boettiger (1957) estimated the strain in the DLMs of a bumblebee Bombus to be approximately $1 \%$ by monitoring the trajectories of light beams reflected off small mirrors affixed to different regions of the thorax. Surholt et al. (1990) used magnetoresistive sensors to measure thoracic oscillations in Bombus terrestris and Xylocopa sulcatipes, and reported strains of 3.5 and $5 \%$ of thorax height in these two species, respectively. Gilmour and Ellington (1993a) used high-speed video recordings to

\footnotetext{
*Author for correspondence (e-mail: flymanmd@violet.berkeley.edu).
} 
track the length changes in a DLM of Bombus lucorum visualized through a window cut in the cuticle, and measured strains ranging from 1.4 to $3 \%$. Molloy (1988) measured the thoracic length changes of a crane fly Tipula at the two stroke reversals within the wingbeat cycle and estimated that the DLMs shortened by $3.5-4 \%$.

Owing to the availability of powerful molecular genetic techniques, the fruit fly Drosophila melanogaster has become an important model system for both muscle biophysics in general and stretch-activation in particular (Peckham et al. 1990; Molloy et al. 1993; Sparrow, 1995; Tohtong et al. 1995). However, there are as yet no measurements of the muscle strain in these small flies during flight. Like all indirect flight muscles, those of Drosophila spp. must generate a considerable amount of mechanical power in accordance with the high cost of flight. Mechanical power estimates for indirect flight muscles in this genus range from approximately 40 to $140 \mathrm{~W} \mathrm{~kg}^{-1}$ muscle mass, based on the metabolic rate of tethered animals (Chadwick and Gilmour, 1953; Kammer and Heinrich, 1978; Laurie-Ahlberg et al. 1985; Götz, 1987; Dickinson and Lighton, 1995). In contrast, values for the power output of isolated skinned fiber preparations vary from approximately 0.02 to $0.08 \mathrm{~W} \mathrm{~kg}^{-1}$ muscle mass, nearly two orders of magnitude smaller than those derived from in vivo measurements (Peckham et al. 1990; Tohtong et al. 1995). This discrepancy is due in part to differences in the physiological milieu between intact and demembranated fibers, and in part to the low strain amplitudes that are required for Nyquist analysis of isolated muscle fibers.

In this study, we have used high-speed video microscopy to monitor displacements of the thoracic sclerites in the fruit fly Drosophila virilis and have estimated the amplitude and spectral characteristics of the strain within both the DLMs and the DVMs. To facilitate our measurements, we used $D$. virilis, which is 1.8 times larger and has a lower wingbeat frequency than D. melanogaster. At $25^{\circ} \mathrm{C}$, the wingbeat frequency of tethered D. virilis is $169 \pm 11 \mathrm{~Hz}(N=9$, mean \pm S.D. $)$, which compares with $208 \pm 15 \mathrm{~Hz}(N=26)$ for D. melanogaster (M. Dickinson, unpublished observations). Despite these differences in frequency, we would expect the muscle strains to be quite similar throughout the genus. The results of this study should provide both a better background for assessing current models of stretch-activation and more accurate strain histories for use in studies of isolated fibers.

\section{Materials and methods}

We used 4-day-old mature females of Drosophila virilis Sturtevant from laboratory cultures maintained at $19^{\circ} \mathrm{C}$. The origin and insertion of all indirect flight muscles are well known from previous anatomical studies (Williams and Williams, 1943; Miller, 1950; Coggshall, 1978). The flies were first immobilized by cooling them to approximately $4^{\circ} \mathrm{C}$ on a Peltier stage (Marlow Industries, Inc., Texas, USA). Small white dots of water-based acrylic paint were then applied to the exoskeleton at a number of origin and insertion points as indicated in Fig. 1A. These white markings provided us with a means of monitoring length changes of the indirect flight muscles on successive video frames. We have followed the nomenclature of Miller (1950) for the individual muscle fibers and that of McAlpine et al. (1981) for the thoracic exoskeleton. The lengths of representative DLM fibers (45c, 45d) were monitored by tracking an anterior mark on the postpronotum (just posterior to the neck) and two posterior marks, one on the anatergite below the scutellum and one on the lateral side of the abdominal tergite. The lengths of two representative DVMs (47a, 47b) were followed by marking above the notopleuron and on the katepisternum. After marking the thorax, we then affixed the fly to the end of a $0.13 \mathrm{~mm}$ diameter tungsten wire tether using an ultraviolet-cured adhesive, Crystal Clear (Loctite, Ohio). In order to examine potential artifacts due to tethering, we mounted flies either dorsally between the head and the thorax or ventrally between the coxae of the meso- and metathoracic legs.

Flight sequences of tethered flies at ambient room temperature $\left(22^{\circ} \mathrm{C}\right)$ were recorded at 3000 frames s$^{-1}$ using a high-speed video system consisting of a Kodak Ektapro EM motion analyzer and image intensifier equipped with an Infinity K2 long-distance microscope and CF-3 objective. The sequence was then downloaded at video rate $\left(30\right.$ frames s$\left.^{-1}\right)$ onto S-VHS tapes. Owing to the high magnification required to obtain reliable measurements at the 3.75:1 frame aspect ratio (240×64 pixels) of the EktaPro at 3000 frames s $^{-1}$, we were constrained to track either the DLMs or the DVMs, but not both simultaneously. Therefore, each of the six flies was videotaped twice, once to record DLM strain and once to record DVM strain. A portion of a representative video sequence is shown in Fig. 1B. Flight sequences were only analyzed if the flies assumed a flight posture similar to that in free flight. Under these conditions, the stroke amplitude is large (approximately $150^{\circ}$ ) and the legs are tucked close to the body and not extended forward as is typical of a landing response. For each of the 12 recorded flight sequences, video sequences of 150 successive frames consisting of 6-8 wingbeat cycles were digitized frame by frame on a Power Macintosh $8100 \mathrm{AV}$ computer and quantified by measuring the distance between the designated landmarks using NIH Image software. The data were subsequently analyzed using Igor (WaveMetrics, Oregon, USA). The corresponding resting lengths of the muscles were calculated from the positions of the origin and insertion landmarks in video sequences taken when the fly was not flying. We calculated the strain as: [(resting length minus observed length)/resting length] $\times 100 \%$. Thus, positive values indicate contraction whereas negative values indicate extension. The spectral compositions of the strains were examined by performing Fast Fourier Transforms (FFTs) on the measured waveforms.

The DLMs and DVMs each contract once in a wingbeat cycle, powering the downstroke and upstroke of the wing, respectively. In order to obtain the maximum length change, we had to maintain a viewing angle perpendicular to the sagittal plane of the fly. With this orientation, however, the 
A

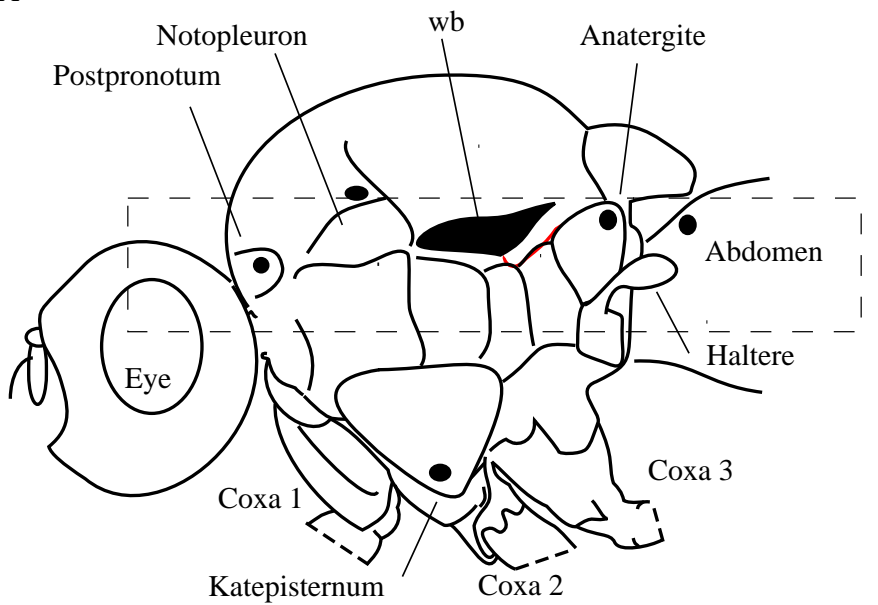

B

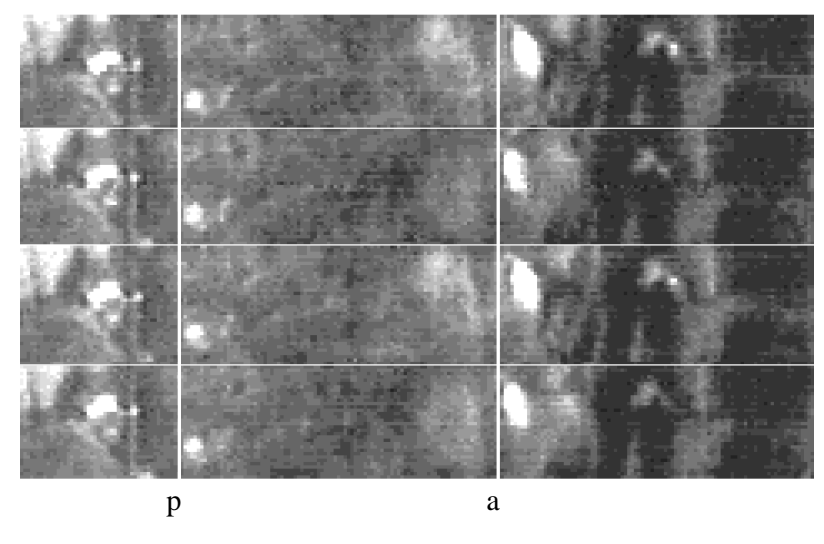

Fig. 1. (A) Schematic diagram of a lateral view of Drosophila virilis showing the position of paint spots (solid black circles) that were applied to track the motion of the origin and insertion sites of the indirect flight muscles. wb, wing base. (B) Four video images of the region indicated by the dashed box in A. The frames correspond to maximum dorsal ventral muscle (DVM) contraction (first and third) and maximum dorsal longitudinal muscle (DLM) contraction (second and fourth) in two successive wingbeat cycles. The images are aligned at the postpronotum (p) to illustrate the magnitude of displacement at the anatergite (a). The white paint spots in B correspond to the black circles in A. Scale bar, $0.5 \mathrm{~mm}$.

haltere on its upstroke obscured the mark on the anatergite during the downstroke of the wing. For this reason, we applied a second mark on the abdominal tergite (Fig. 1A) to allow the positions of these missing points to be estimated. This correction was implemented by first calculating the correlation between the postpronotum-to-anatergite distances and the postpronotum-to-abdominal tergite distances from all the video frames in which the three applied landmarks were visible. The resulting correlation coefficients were then used to interpolate the missing postpronotum-to-anatergite distances in frames in which the haltere obscured the position of the anatergite. Although there was some relative motion between the thorax and abdomen during flight, the correlation (mean $r^{2}=0.72 \pm 0.070$, mean \pm S.D., $N=6$ ) was adequate for the interpolation.

\section{Results}

The cyclical activity of the muscles is evident in the 150frame flight sequences shown in Fig. 2. We measured the difference between the maximum and minimum strain within each cycle to determine the peak-to-peak strain amplitude. In order to test whether the strain amplitude of muscle contraction was altered by the tethering procedure, we tethered flies in two different locations. We observed no statistical differences in the amplitude or frequency of the thoracic strains between the flies tethered dorsally at the notum or ventrally at the coxae $(P>0.05$, two-tailed $t$-test). We pooled the data recorded from the dorsally and ventrally tethered flies to calculate the mean values of muscle strain.

The observed mean strain amplitude and the magnitude of the peaks at the dominant frequencies in six flies are summarized in Table 1. The observed strain amplitudes were $3.5 \pm 0.70 \%$ (S.D.) for the DLMs and $3.3 \pm 0.40 \%$ for the DVMs. The strain amplitudes of the DLMs and DVMs were quite similar although, as explained above, the two muscles were not digitized from the same flight sequence since the narrow aspect ratio of the Ektapro frame made it difficult to visualize all four cuticle marks in the same frame with adequate resolution. However, in one instance (for fly 5) where we were able to monitor both muscles in the same flight sequence, we observed a similar average strain amplitude with the expected $180^{\circ}$ phase shift (Fig. 2C). The wingbeat frequency for each fly was estimated from the number of wingbeat cycles within the 150frame video sequence.

The average mean oscillating lengths of both the DLMs and DVMs were not significantly different from the measured resting lengths (Table 1; Fig. 2). However, in two preparations, the muscles oscillated around a mean length that was $1 \%$ or more shorter than the resting length (Table 1: fly 3 DLM, $1.4 \%$; fly 6 DVM, $1 \%$ ). Thus, in these cases, the muscles were contracted with respect to the resting length during most of the wingbeat cycle. Deviations of this magnitude are unlikely to result from digitization error and might be caused either by differences in the recruitment of the antagonist flight muscles or through the action of direct control muscles that have the ability to change the resonance mechanics of the thorax. In either case, offsets of this magnitude might have a small but significant effect on mechanical power output. In the green crab Carcinus maenas, the work output of the scaphognathite muscle drops by approximately $20 \%$ when the mean oscillatory length is changed by $\pm 5 \%$ of the optimal length (Josephson and Stokes, 1989). Although the scaphognathite muscle is a synchronous 


\section{W. P. Chan AND M. H. Dickinson}

Table 1. A summary of the observed mean strain and oscillation amplitude of the indirect flight muscles during flight

\begin{tabular}{|c|c|c|c|c|c|c|c|}
\hline & Fly & Mean strain $(\%)$ & Strain amplitude $(\%)$ & Fundamental (\%) & First harmonic (\%) & Wingbeat frequency $(\mathrm{Hz})$ & $N$ \\
\hline \multirow[t]{5}{*}{ DLM } & 1 & 0.8 & $4.6 \pm 0.32$ & 3.1 & 1 & 165 & 7 \\
\hline & 2 & 0.7 & $4.1 \pm 0.32$ & 2.2 & 0.4 & 154 & 7 \\
\hline & 4 & 0.0 & $2.9 \pm 0.14$ & 0.9 & 0.1 & 173 & 8 \\
\hline & 5 & 0.4 & $3.2 \pm 0.58$ & 1.1 & 0.1 & 168 & 7 \\
\hline & 6 & -0.3 & $3.2 \pm 0.51$ & 2.3 & 0.2 & 140 & 6 \\
\hline \multirow[t]{6}{*}{ DVM } & 1 & -0.3 & $3.0 \pm 0.31$ & 2 & 0.6 & 189 & 9 \\
\hline & 2 & 0.5 & $3.5 \pm 0.47$ & 2.5 & 0.1 & 153 & 7 \\
\hline & 3 & 0.2 & $3.2 \pm 0.29$ & 2.3 & 0.2 & 163 & 7 \\
\hline & 4 & -0.4 & $3.7 \pm 0.33$ & 1.2 & 0.3 & 178 & 8 \\
\hline & 5 & -0.2 & $3.6 \pm 0.38$ & 1.9 & 0.1 & 152 & 7 \\
\hline & 6 & 1.0 & $2.7 \pm 0.43$ & 1.5 & 0 & 159 & 7 \\
\hline
\end{tabular}

Strain amplitude (mean \pm S.D.) was measured from the peak values of each cycle within the 150 -frame video sequences.

The magnitude of the fundamental and the first harmonic of the power spectrum for each waveform are also indicated.

The wingbeat frequency for each fly was estimated from the number of complete wingbeat cycles $(N)$ within each sequence.

Flies 1-3 were attached dorsally, flies 4-6 were attached ventrally.

Activities of the DLM and DVM for each fly were recorded separately.

muscle, the mechanical power of asynchronous muscles undergoing large strain is also likely to depend on mean length.

The spectral composition of the strain waveforms includes significant energy at higher harmonics, indicating that the oscillations in muscle length are not simply sinusoidal (Figs 2, $3)$. In order to compare across preparations, we normalized the spectra to the fundamental frequency. The spectral composition did not appear to vary according to the tethering

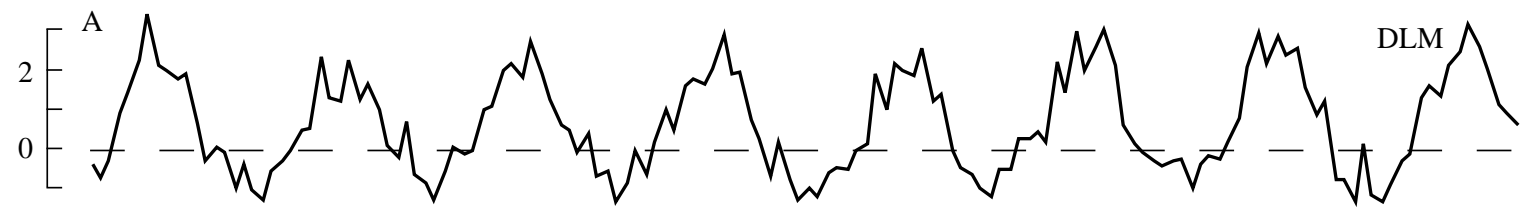

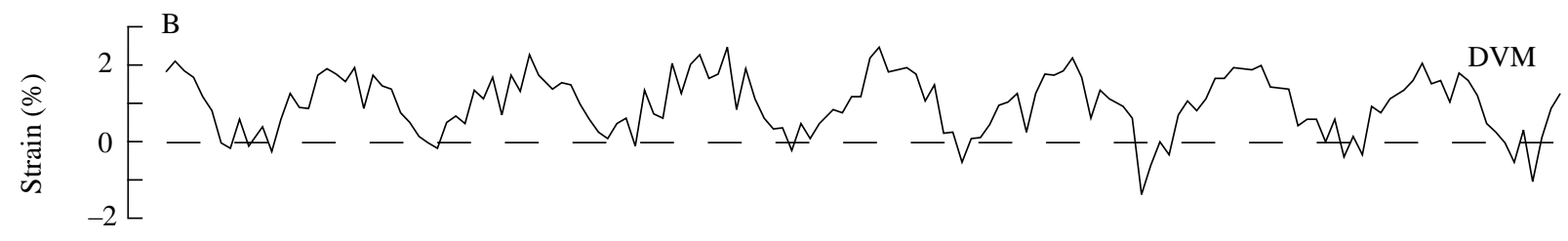

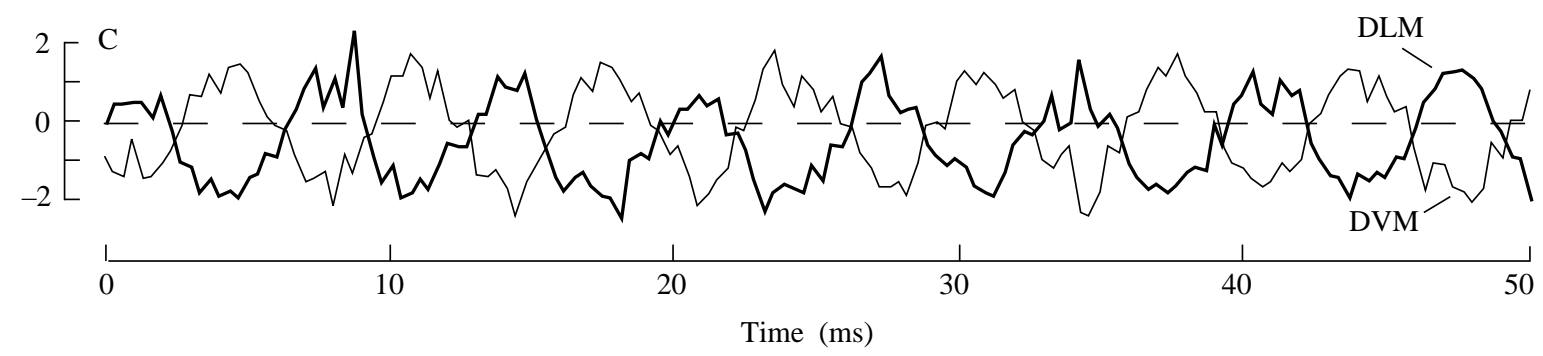

Fig. 2. The activity of the indirect flight muscles over several wingbeat cycles. (A) Strain amplitude of the DLMs from fly 2. (B) Strain amplitude of the DVMs from fly 6. The mean strain is greater than 0. (C) For fly 5, we were able to track both the DLMs and the DVMs in the same frame with adequate spatial resolution. The strain amplitudes of the two antagonists are similar but in antiphase to each other. The dashed lines in all panels represent the resting length (zero strain) of the muscles. 


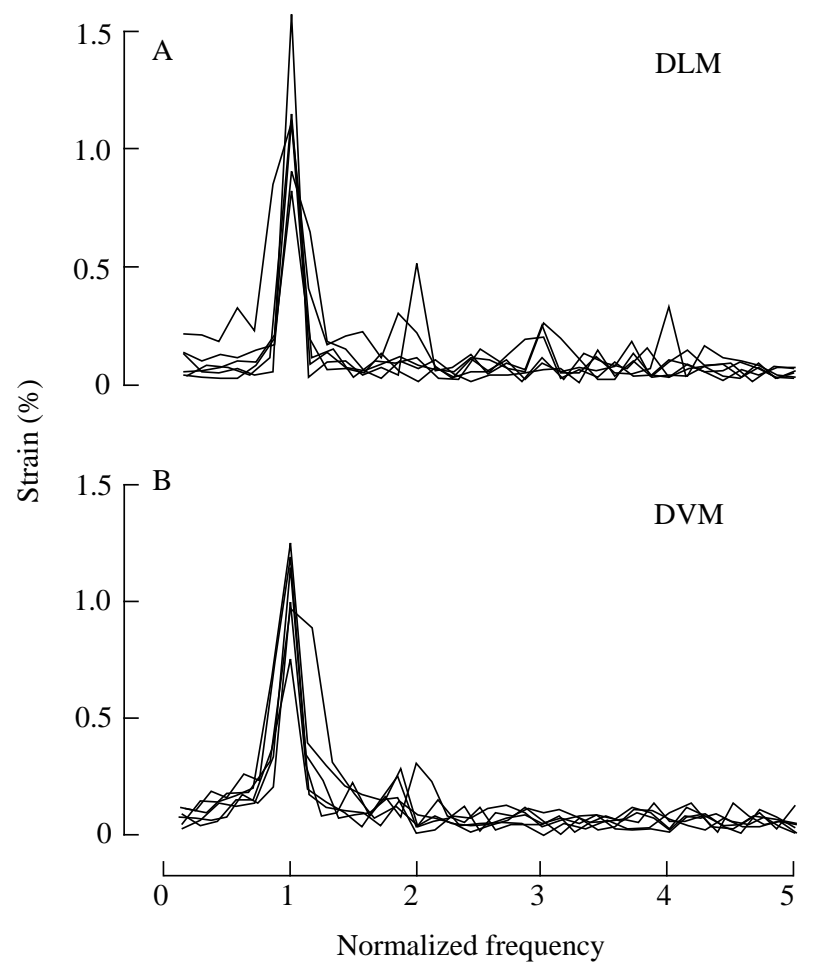

Fig. 3. Normalized Fourier transforms of the observed strain amplitude in the DLMs (A) and the DVMs (B) of six flies. Spectra are normalized to the fundamental frequency. The power spectra from flies tethered dorsally or ventrally are similar and there is a considerable amount of energy in the higher harmonics

position (Table 1), again suggesting that our attachment procedure did not drastically compromise the measurements of muscle strain. The spectral composition appears comparable to that reported by Gilmour and Ellington (1993a) for Bombus lucorum. There is some indication that the higher harmonics are stronger in the oscillations of the DLMs than of the DVMs, although this difference was not statistically significant (assessed by comparing the difference in the ratios of the fundamental and second harmonic; $P>0.05$, one-tailed $t$-test).

\section{Discussion \\ Strain amplitude}

By using high-speed video microscopy we have provided the first estimates of the in vivo strain of the indirect flight muscles of the fruit fly Drosophila virilis. In our study, the mean strain amplitudes observed for the DLMs and the DVMs were 3.5 and $3.3 \%$ of the resting length, respectively. These values fall within the range of measurements from other species using a variety of methods (Boettiger, 1957; Molloy, 1988; Surholt et al. 1990; Gilmour and Ellington, 1993a). Because of the constraints imposed by working with animals as small as Drosophila, we were unable to implement the more accurate windowing technique employed by Gilmour and Ellington (1993a) and, instead, monitored the changes in
In vivo length changes in fruit fly flight muscle

muscle length less invasively, through the motion of muscle origin and insertion points on the thorax. The indirect flight muscles of Drosophila virilis are similar to those of many arthropod muscles in that they insert directly onto the internal surface of the cuticle via modified Z-bands on the underlying epithelial cells, without the presence of an intervening tendonous apodeme (Auber, 1963; Shafiq, 1964; Lai-Fook, 1967). Therefore, measuring muscle length by tracking the position of origins and insertions is not likely to result in significant overestimates of actual sarcomere length changes.

The wing-stroke amplitude of flying Drosophila spp. correlates both with total flight force (Götz et al. 1979; Götz and Wandel, 1984) and with mechanical power output (Dickinson and Lighton, 1995). It is thus reasonable to suppose that the largest muscle strains would occur during times of maximum force production. In the absence of elaborate visual feedback, tethered fruit flies may not produce all the flight force required to sustain hovering. Measurements of the total flight force of tethered animals in the absence of closed-loop visual feedback are as varied as $41 \%$ (Götz, 1968), $75 \%$ (Lehmann, 1994) and 100\% (Dickinson and Götz, 1996). Since we did not provide any visual feedback in these experiments, our estimates of muscle strain amplitude must be considered as a lower limit.

\section{Strain and power output of indirect flight muscle}

In terms of energy required per unit time, flight is the costliest form of locomotion. Accordingly, the indirect flight muscles of insects exhibit many specializations, including stretch-activation, that enable them to sustain high levels of mechanical power output. The power output of Drosophila spp. flight muscle has been estimated by measuring sugar and $\mathrm{O}_{2}$ consumption as well as $\mathrm{CO}_{2}$ production, with values ranging from approximately 40 to $140 \mathrm{~W} \mathrm{~kg}^{-1}$ muscle mass (Chadwick and Gilmour, 1953; Kammer and Heinrich, 1978; Laurie-Ahlberg et al. 1985; Götz, 1987; Dickinson and Lighton, 1995). Even the lowest of these in vivo estimates are over two orders of magnitude larger than the maximum power reported from Nyquist analysis of skinned fibers (Peckham et al. 1990; Tohtong et al. 1995). There is probably no single reason for this discrepancy, and the power output of skinned insect muscle fibers rarely approaches that measured in intact membranated muscles. However, the values of muscle strain used in the skinned fiber preparations $(0.25-0.5 \%)$ are much smaller than those reported here (3.3-3.5\%), which might partially explain the low power output of the in vitro preparations. Nyquist analysis is a powerful technique designed to investigate the mechanical properties of the contractile apparatus, but requires low imposed strain in order to obtain a linear response. Higher power output can be obtained with larger strain amplitudes. For example, Gilmour and Ellington (1993b) found that skinned bee Bombus lucorum muscle could generate a power output as great as $110 \mathrm{~W} \mathrm{~kg}^{-1}$ muscle mass, but only at strains as high as $4-5 \%$. 


\section{Implications for crossbridge dynamics}

Strain amplitude is a critical parameter in any attempt to determine the actual motion of the actin and myosin crossbridges in stretch-activated muscle. The sarcomere length of the DLM in Drosophila melanogaster is $3.4 \mu \mathrm{m}$ (Miller et al. 1993). Assuming that the sarcomeres of the indirect flight muscle in D. melanogaster and $D$. virilis are of similar length, a strain of $3.5 \%$ will correspond to a displacement of $60 \mathrm{~nm}$ for each half-sarcomere. However, not all of this strain is necessarily relieved at the crossbridges themselves because of the serial compliances represented by the actin filaments (Julian and Morgan, 1981; Bagni et al. 1990; Kojima et al. 1994; Higuchi et al. 1995), myosin rods (Haselgrove, 1975; Huxley et al. 1994) and C-filaments (Granzier et al. 1996). Wakabayashi et al. (1994) have calculated that actin and myosin filaments could contribute to as much as $70 \%$ of the compliance of the sarcomere in frog skeletal muscle. If such high filament compliance were also true of Drosophila spp. indirect flight muscle, then the myosin heads would move by approximately $18 \mathrm{~nm}$ relative to their thin filament attachment sites from maximum extension to maximum shortening. Estimates for the working stroke or 'throw' of single crossbridges in experiments with dissociated actin and myosin molecules vary from $4 \mathrm{~nm}$ (rabbit psoas muscle, Molloy et al. 1995 ) to approximately $10 \mathrm{~nm}$ (rabbit skeletal muscle, Finer $e t$ al. 1994; Ishijima et al. 1994; chicken pectoralis muscle, VanBuren et al. 1995). In more recent experiments using a regularly oriented array of rabbit skeletal muscle myosin molecules that more accurately mimics the endogenous arrangement, Ishijima et al. (1996) obtained a larger estimate of $20 \mathrm{~nm}$. This value is comparable to the in vivo crossbridge strains reported here, assuming that the serial compliances are as high as in frog skeletal muscle (Wakabayashi et al. 1994). This would suggest that some individual actin and myosin crossbridges might remain attached throughout an entire contraction cycle. However, the helical arrangement of myosin heads and their binding sites on thin filaments is unlikely to allow a perfect spatial alignment of all active crossbridges. In activated skinned rabbit psoas fibers, as much as $39 \%$ of the crossbridges are in the detached state (Kawai and Halvorson, 1991). Even when the change in half-sarcomere length is equivalent to the single myosin throw, many crossbridges must break and form within each cycle. For example, as the halfsarcomere contracts (or extends) by $20 \mathrm{~nm}$, crossbridges already near the limit of their throw must detach. By the same token, the change in half-sarcomere length may bring unattached myosin heads closer to binding sites, thereby initiating the formation of new crossbridges. Nevertheless, our measurements suggest that individual myosin heads may not need to ratchet repetitively along the thin filaments within each contraction cycle.

A recent model of stretch-activation proposes the existence of a short tether formed by the N-terminal extension of the myosin regulatory light chain (MLC2) (Tohtong et al. 1995). Upon stretch, the tethers might function to bring the myosin heads in close opposition to their thin filament binding sites, thereby causing delayed activation by increasing the number of active crossbridges. Tohtong et al. (1995) have also shown that serine to alanine substitutions at two phosphorylation sites of MLC2 significantly attenuate stretch-activation in skinned D. melanogaster DLMs, without affecting $\mathrm{Ca}^{2+}$ activation. This sensitivity of stretch activation to the phosphorylation sites of MLC2 might result from an impairment of the Nterminal tethers. If the crossbridge strains are as small as $20 \mathrm{~nm}$, then it is feasible that the putative N-terminal tethers of MLC2 could remain attached throughout the entire contraction cycle.

There are two important caveats to the above conclusions. First, if the thick and thin filaments are not as compliant as they are in frog muscle, then more of the $60 \mathrm{~nm}$ change in halfsarcomere length will occur at the level of the crossbridges. Second, in a few cases, we measured muscle strains larger than $5 \%$ which, even with serial compliance, would result in strains as great as $26 \mathrm{~nm}$. Under these higher strain amplitudes, it would seem unlikely that any individual crossbridge could remain attached throughout the contraction cycle. In addition, molecular tethers running in parallel with the crossbridges must either be long enough to accommodate $60 \mathrm{~nm}$ strain without breaking or possess the ability to slide along the thin filaments.

We would like to thank Dr David Maughan for the helpful discussion that inspired this study. This work was funded in part by the David and Lucille Packard Foundation and NSF Grant IBN-9208765 to M.H.D.

\section{References}

AIDLEY, D. J. (1985). Muscular contraction. In Comprehensive Insect Physiology, Biochemistry and Pharmacology, vol. 5 (ed. G. A. Kerkut and L. I. Gilbert), pp. 407-437. New York: Pergamon Press. AleXANDER, R. McN. (1995). Springs for wings. Nature 268, 50-51.

AuBER, J. (1963). Ultrastructure de la junction myo-epidermique chez les Dipteres. J. Microscopie 2, 325-336.

Bagni, M. A., Cecchi, G., Colomo, F. and Poggesi, C. (1990). Tension and stiffness of frog muscle fibres at full filament overlap. J. Muscle Res. Cell Motil. 11, 371-377.

Boettiger, E. G. (1957). The machinery of insect flight. In Recent Advances in Invertebrate Physiology (ed. B. T. Scheer), pp. 117-142. Eugene: University of Oregon Publications.

Boettiger, E. G. ANd Furshran, E. (1952). The mechanics of flight movements in Diptera. Biol. Bull. mar. biol. Lab., Woods Hole 102, 200-211.

Chadwick, L. E. AND GILmour, D. (1953). Respiration during flight in Drosophila repleta Wollaston: The oxygen consumption considered in relation to wing-rate. Physiol. Zool. 13, 398-410.

Coggshall, J. C. (1978). Neurons associated with the dorsal longitudinal flight muscles of Drosophila melanogaster. J. comp. Neurol. 177, 707-720.

Dickinson, M. H. AND Götz, K. G. (1996). The wake dynamics and flight forces of the fruit fly Drosophila melanogaster. J. exp. Biol. 199, 2085-2104. 
Dickinson, M. H. And Lighton, J. R. B. (1995). Muscle efficiency and elastic storage in the flight motor of Drosophila. Science 128, 87-89.

Dickinson, M. H. And Tu, M. S. (1996). The function of Dipteran flight muscle. Comp. Biochem. Physiol. A (in press).

Ellington, C. P. (1991). Limitations on animal flight performance. J. exp. Biol. 160, 71-91.

EnNos, R. (1987). A comparative study of the flight mechanism of Diptera. J. exp. Biol. 127, 355-373.

Finer, J. T., Simmons, R. M. And Spudich, J. A. (1994). Single myosin molecule mechanics: piconewton forces and nanometer steps. Nature 368, 113-119.

Gilmour, K. M. And Ellington, C. P. (1993a). In vivo muscle length changes in bumblebees and the in vitro effects on work and power. J. exp. Biol. 183, 101-113.

Gilmour, K. M. And Ellington, C. P. (1993b). Power output of glycerinated bumblebee flight muscle. J. exp. Biol. 183, 77-100.

GöTZ, K. G. (1968). Flight control in Drosophila by visual perception of motion. Kybernetic 4, 199-208.

GöTZ, K. G. (1987). Course-control, metabolism and wing interference during ultralong tethered flight in Drosophila melanogaster. J. exp. Biol. 128, 35-46.

Götz, K. G., Hengstenberg, B. And Biesinger, R. (1979). Optomotor control of wing beat and body posture in Drosophila. Biol. Cybernetics 35, 101-112.

GöTZ, K. G. AND WANDEL, U. (1984). Optomotor control of the force of flight in Drosophila and Musca. II. Covariance of lift and thrust in still air. Biol. Cybernetics 51, 135-139.

Granzier, H., Helmes, M. And Trombitás, K. (1996). Nonuniform elasticity of titin in cardiac myocytes: a study using immunoelectron microscopy and cellular mechanics. Biophys. J. 70, 430-442.

Haselgrove, J. C. (1975). X-ray evidence for conformational changes in the myosin filaments of vertebrate striated muscle. $J$. molec. Biol. 92, 113-143.

HeIDE, G. (1983). Neural mechanisms of flight contol in Diptera. In BIONA-Report 2 (ed. W. Nachtigall), pp. 35-52. Stuttgart: Fischer.

Higuchi, H., YANAGIDA, T. AND Goldman, Y. E. (1995). Compliance of thin filaments in the skinned fibers of rabbit skeletal muscle. Biophys. J. 69, 1000-1010.

Huxley, H. E., Stewart, A., Sosa, H. And Irving, T. (1994). X-ray diffraction measurements of the extensibility of actin and myosin filaments in contracting muscle. Biophys. J. 67, 2411-2421.

Ishijima, A., Harada, Y., Kojima, H., Funatsu, T., Higuchi, H. And YANAGIDA, T. (1994). Single-molecule analysis of the actomyosin motor using nano-manipulation. Biochem. biophys. Res. Commun. 199, 1057-1063.

Ishijima, A., Kojima, H., Higuchi, H., Harada, Y., Funatsu, T. AND YANAGIDA, T. (1996). Multiple- and single-molecule analysis of the actomyosin motor by nanometer-piconewton manipulation with a microneedle: unitary steps and forces. Biophys. J. 70, 383-400.

Josephson, R. K. And Stokes, D. R. (1989). Strain, muscle length and work output in a crab muscle. J. exp. Biol. 145, 45-61.

JosephSON, R. K. AND Young, D. (1987). Fiber ultrastructure and contraction kinetics in insect fast muscles. Am. Zool. 27, 991-1000.

Julian, F. J. AND MoRgan, D. L. (1981). Tension, stiffness, unloaded shortening speed and potentiation of frog muscle fibres at sarcomere lengths below optimum. J. Physiol., Lond. 319, 205-217.

KAMmer, A. E. (1985). Flying. In Comprehensive Insect Physiology,
Biochemistry and Pharmacology, vol. 5 (ed. G. A. Kerkut and L. I. Gilbert), pp. 491-552. New York: Pergamon Press.

KAMmer, A. E. AND HeInRICH, B. (1978). Insect flight metabolism. Adv. Insect Physiol. 13, 133-228.

Kawai, M. And Halvorson, H. R. (1991). Two step mechanism of phosphate release and the mechanism of force generation in chemically skinned fibers of rabbit psoas muscle. Biophys. J. 59, 329-342.

KojIma, H., IshiJima, A. And Yanagida, T. (1994). Direct measurement of stiffness of single actin filaments with and without tropomyosin by in vitro nanomanipulation. Proc. natn. Acad. Sci. U.S.A. 91, 12962-12966.

LAI-FoOK, J. (1967). The structure of developing muscle insertions in insects. J. Morph. 123, 503-528.

Laurie-Ahlberg, C. C., Barnes, P. T., Curtsinger, J. W., Emigh, T. H., Karlin, B., Morris, R., Norman, R. A. And Wilton, A. N. (1985). Genetic variability of flight metabolism in Drosophila melanogaster. II. Relationship between power output and enzyme activity levels. Genetics 111, 845-868.

LehmanN, F.-O. (1994). Aerodynamische, kinematische und electrophysiologische Aspekte der Flugkrafterzeugung und Flugkraftsteuerung bei der Taufliege Drosophila melanogaster. $\mathrm{PhD}$ dissertation, Eberhad-Karls-Universität, Tübingen.

Machin, K. E. And PRIngle, J. W. S. (1960). The physiology of insect fibrillar muscle. III. The effect of sinusoidal changes of length on a beetle flight muscle. Proc. R. Soc. Lond. B 152, 311-330.

McAlpine, J. F., Peterson, B. V., Shewell, G. E., Teskey, H. J., Vockeroth, J. R. AND Wood, D. M. (1981). Manual of Nearctic Diptera. Research Branch, Agriculture Canada.

Miller, A. (1950). The internal anatomy and histology of the imago of Drosophila melanogaster. In Biology of Drosophila (ed. M. Demerec), pp. 420-534. New York: John Wiley.

Miller, R. C., SchaAf, R., Maughan, D. W. and Tansey, T. R. (1993). A non-flight muscle isoform of Drosophila tropomyosin rescues an indirect flight muscle tropomyosin mutant. J. Muscle Res. Cell Motil. 14, 85-98.

MiYan, J. A. AND EwING, A. W. (1985). How Diptera move their wings: a re-examination of the wing base articulation and muscle systems concerned with flight. Phil. Trans. R. Soc. Lond. B 311, 271-302.

Molloy, J. E. (1988). Active insect fibrillar flight muscle, its mechanical performance and cross-bridge kinetics. $\mathrm{PhD}$ thesis, University of York.

Molloy, J. E., Burns, J. E., Kendrick-Jones, J., TregeAr, R. T. And White, D. C. S. (1995). Movement and force produced by a single myosin head. Nature 378, 209-212.

Molloy, J., Kreuz, A., Miller, R., Tansey, T. and Maughan, D. (1993). Effects of tropomyosin deficiency in flight muscle of Drosophila melanogaster. In Mechanism of Myofilament Sliding in Muscle Contraction (ed. H. Sugi and G. H. Pollack), pp. 165-172. New York: Plenum Press.

Peckham, M., Molloy, J. E., Sparrow, J. C. and White, D. C. S. (1990). Physiological properties of the dorsal longitudinal flight muscle and the tergal depressor of the trochanter muscle of Drosophila melanogaster. J. Muscle Res. Cell Motil. 11, 203-215.

Pringle, J. W. S. (1949). The excitation and contraction of the flight muscles of insects. J. Physiol., Lond. 108, 226-232.

Pringle, J. W. S. (1957). Insect Flight. Cambridge: Cambridge University Press.

RoEDER, K. (1951). Movements of the thorax and potential changes 


\section{W. P. Chan and M. H. Dickinson}

in the thoracic muscles of insects during flight. Biol. Bull. mar. biol. Lab., Woods Hole 100, 95-106.

SHAFIQ, S. A. (1964). An electron microscopical study of the innervation and sarcoplasmic reticulum of the fibrillar flight muscle in Drosophila melanogaster. Q. J. microsc. Sci. 105, 1-6.

SNODGRASS, R. E. (1993). Principles of Insect Morphology. Ithaca: Cornell University Press.

Sotavalta, O. (1947). The flight-tone (wing stroke frequency) of insects. Acta ent. Fenn. 4, 1-117.

Sparrow, J. C. (1995). Flight and phosphorylation. Nature 374, 592-593.

Surholt, B., Greive, H., BaAl, T. and Bertsch, A. (1990). Nonshivering thermogenesis in asynchronous flight muscles of bumblebees? Comparative studies on males of Bombus terrestris, Xylocopa sulcatipes and Acherontia atropos. Comp. Biochem. Physiol. 97A, 493-499.

Tohtong, R., Yamashita, H., Graham, M., Haeberle, J., Simcox, A. AND Maughan, D. (1995). Impairment of muscle function caused by mutations of phosphorylation sites in myosin regulatory light chain. Nature 374, 650-653.

VanBuren, P., Guilford, W. H., Kennedy, G., Wu, J. And Warshaw, D. M. (1995). Smooth muscle myosin: a high forcegenerating molecular motor. Biophys. J. 68, 256s-259s.

WaKabayashi, K., Sugimoto, Y., TanaKa, H., Ueno, Y., TakeZawa, Y. AND AMEMIYA, Y. (1994). X-ray diffraction evidence for the extensibility of actin and myosin filaments during muscle contraction. Biophys. J. 67, 2422-2435.

Williams, C. M. AND Williams, M. V. (1943). The flight muscles of Drosophila repleta. J. Morph. 72, 589-599.

WISSER, A. (1988). Wing beat of Calliphora erythrocephala: turning axis and gearbox of the wing base (Insecta, Diptera). Zoomorphology 107, 359-369.

Wisser, A. AND Nachtigall, W. (1984). Functional-morphological investigations on the flight muscles and their insertion points in the blowfly Calliphora erythrocephala (Insecta, Diptera). Zoomorphology 104, 188-195. 